\title{
ESTUDIO MORFOESTRUCTURAL DE UNA POBLACIÓN DE CERDOS NATURALIZADOS EN LOS CANTONES VALENCIA Y LA MANÁ, ECUADOR
}

\author{
${ }^{\bullet}$ Kléber Estupiñán Véliz ${ }^{1,2}$, Diana Vasco Mora $^{2}$, Sara Barreto ${ }^{2}$ y Kléber Zambrano ${ }^{2}$ \\ ${ }^{1}$ Unidad de Investigación Científica y Tecnológica, Universidad Técnica Estatal de Quevedo, $\mathrm{km} 1$ 1/2 vía \\ Quevedo-Santo Domingo de los Tsáchilas, C.P. 73. Quevedo, Los Ríos, Ecuador. ${ }^{k} k 1$ stu2004audy@yahoo.com \\ ${ }^{2}$ Facultad de Ciencias Pecuarias, Universidad Técnica Estatal de Quevedo, $\mathrm{km} 7$ vía a El Empalme \\ Mocache, Los Ríos, Ecuador
}

RESUMEN

E l estudio se realizó en una muestra de 149 cerdos naturalizados de 12 a 36 meses de edad, de los cuales 61 pertenecían a Valencia y 88 a La Maná. Se efectuó la caracterización morfológica de 16 variables zoométricas, ocho fanerópticas y se calcularon nueve índices zoométricos. Las principales variables fueron: alzada a la cruz (ALC), diámetro longitudinal (DL), perímetro torácico (PTO), longitud de grupa (LGR), ancho de grupa (AGR), obteniéndose los siguientes promedios: ALC 60.99 y $61.74 \mathrm{~cm}$; DL 80.12 y $79.12 \mathrm{~cm}$; PTO 93.68 y $92.50 \mathrm{~cm}$; LGR 30.08 y $28.32 \mathrm{~cm}$ y, AGR 23.08 y 22.26 $\mathrm{cm}$; para Valencia y La Maná, respectivamente. El índice cefálico fue de $45.89 \%$ en Valencia y $51.15 \%$ en La Maná. En los cerdos naturalizados se pudo observar que en mayor porcentaje presentaron el perfil frontonasal recto; el color de capa que se registró con mayor frecuencia fue colorada en Valencia y manchada en La Maná; en el color de la mucosa sobresale la oscura en Valencia y clara en La Maná; el pelaje va de abundante a escaso; se evidenciaron en mayor cantidad cerdos con orejas tipo tejas en ambas localidades; se encontró poca población con mamellas y prevaleció el cerdo doble propósito en Valencia y magro en La Maná.

Palabras claves: Zoométrica, faneróptica, índice zoométrico.

Abstract

$\mathrm{T}$ he study was carried out sampling 149 naturalized pigs with an age range from 12 to 36 months, from this group 61 belonged to Valencia and 88 to La Maná. The morphologic characterization of 16 zoometric and eight phaneroptical variables was made and nine zoometrics indexes were calculated. The main variables analized were: raised to the cross (CRS), longitudinal diameter (LD), thoracic perimeter (TP), croup length (CL), croup width (CW) obtaining the following averages: CRS 60.99 and $61.74 \mathrm{~cm}$; LD 80.12 and $79.12 \mathrm{~cm}$; TP 93.68 and $92.50 \mathrm{~cm}$; CL 30.08 and $28.32 \mathrm{~cm}$ and, CW 23.08 and $22.26 \mathrm{~cm}$; for Valencia and La Maná, respectively. The cephalic index was of $45.89 \%$ in Valencia and $51.15 \%$ in La Maná. The naturalized pigs showed in higher percentage the rectilinear frontonasal profile; the most frequent layer color was colored in Valencia and dappled in La Maná; the color of the mucous is mainly dark in Valencia and light in La Maná; the pelage varies from abundant to scarce; more pigs with Iberian ears types have been found in both towns; a reduced number of animals with mamellas was registered while the pig of double purpose prevailed in Valencia and meager in La Maná.

Key words: Zoometric, phaneroptical, zoometric index.

\section{INTRODUCCIÓN}

$\mathrm{L}$ a caracterización morfológica y faneróptica es base fundamental para el conocimiento de la producción animal, desde el punto de vista de la conservación de los recursos genéticos, por tanto, es necesaria para la definición, descripción, tipificación y diferenciación de poblaciones (Barba et al., 2004). Los cerdos criollos del Caribe y Latinoamérica están fuertemente ligados a la explotación familiar en culturas locales amenazadas por la transculturación. Según Delgado et al. (2004) en la actualidad existe un creciente interés científico en las razas locales criollas con grandes posibilidades para la diversificación de la producción porcina en la búsqueda de productos de calidad.

La inclusión de los genotipos locales en los procesos comerciales de producción es una opción atractiva para su conservación, pero requiere de una adecuada ca-

Recibido: Junio, 2008. Aceptado: Octubre, 2008.

Publicado como ARTÍCULO en Ciencia y Tecnología 2(2): 15-20. 2009 racterización previa. Difícilmente se puede considerar valioso un recurso que no ha sido caracterizado. Para aprovecharlos adecuadamente deben ser caracterizados y establecer estrategias para conservarlos (Revidatti et al., 2004). El cerdo criollo ecuatoriano, como parte de esa variabilidad genética, es una especie poco conocida, analizada y valorada en el territorio nacional y que se ha visto desplazado por la introducción de razas mejoradas en las explotaciones pecuarias (Estupiñán et al., 2007).

Del cerdo criollo se conoce poco, se sabe que su ancestro es el cerdo ibérico, se le han atribuido ventajas como la rusticidad, capacidad para el consumo de alimentos altos en fibra y otros, que no están avaladas totalmente por la evidencia experimental. Por otra parte, la caracterización de su comportamiento tanto reproductivo como de crecimiento y canal es bastante limitado. En España, su país de origen, existen diferentes variedades de cerdos ibéricos que tienen en común tres aspectos fundamentales: producción de alimentos de 
alta calidad (jamón de cerdo ibérico), adaptación a las condiciones de producción extensivas de la dehesa mediterránea y variabilidad genética y rusticidad (Delgado et. al., 1998).

En estudios de las variables e índices zoométricos, realizados en Cuba en cerdos hembras y machos, se aprecia mayor nivel en los machos para todas las variables morfológicas. Reportando alzada de la cruz de 67.30 y $61.37 \mathrm{~cm}$; alzada de la grupa de 73.09 y 66.40 $\mathrm{cm}$; el diámetro longitudinal de 76.49 y $71.70 \mathrm{~cm}$, para hembras y machos, respectivamente (Barba et al., 1998). En trabajos efectuados por Sanz et al. (2004) en cerdos de seis variedades dentro del tronco ibérico, se reporta alzada de la cruz de: 77.00; 77.40; 76.40; 77.40; 77.40 y $78.10 \mathrm{~cm}$; y, alzada de la grupa de $83.00 ; 79.90 ; 80.90$; $81.00 ; 81.70$ y $81.30 \mathrm{~cm}$, para las variedades Mamellada, Entrepelada, Retinto, Lampiño, Silvela y Tobiscal, respectivamente.

En la caracterización realizada por Revidatti et al. (2005) con una población de cerdos en la provincia de Corrientes, Argentina se registran pesos promedio de $60.47 \mathrm{~kg}$ con CV de 41\%; alzada a la cruz de $56.09 \mathrm{~cm}$ y perímetro torácico de $94.29 \mathrm{~cm}$. Hurtado et al. (2005) reporta valores de $59.51 \mathrm{~cm}$ para alzada a la cruz, 74.69 $\mathrm{cm}$ de diámetro longitudinal y $84.85 \mathrm{~cm}$ de perímetro torácico en el estudio morfológico del cerdo criollo del estado de Apure en Venezuela.

En esta investigación se planteó conocer el material genético existente en los cantones "Valencia" y "La Maná", lo que permitirá a mediano y largo plazo seleccionar animales de gran potencial mediante la caracterización morfoestructural y faneróptica de cerdos naturalizados.

\section{Materiales y MÉtodos}

$\mathrm{L}$ a investigación se realizó en los cantones Valencia $\perp_{y}$ La Maná, provincias de Los Ríos y Cotopaxi, respectivamente. La muestra aleatoria de la población de cerdos naturalizados estuvo compuesta de 149 ejemplares, de 12 a 36 meses de edad, de los cuales 61 pertenecían a Valencia y 88 a La Maná. Se utilizó compás de broca, bastón zoométrico, balanza de gancho de $200 \mathrm{~kg}$ de capacidad y cinta métrica inextensible.

Se midieron 16 variables zoométricas propuestas por Aparicio (1960); Sotillo (1985) y Barba et al. (2004): longitud de cabeza (LCZ), anchura de cabeza (ACZ), longitud de hocico (LH), anchura de hocico (AH), longitud de grupa (LGR), anchura de grupa (AGR), alzada a la cruz (ALC), alzada a la grupa (ALG), alzada al nacimiento de la cola (ANC), diámetro longitudinal (DL), diámetro dorsoesternal (DDE), diámetro bicostal (DBC), perímetro toráxico (PTO), perímetro de la caña (PCA), longitud de oreja (LO) y anchura de oreja (AO). Con la información obtenida se calcularon nueve índices zoométricos, resultado de la combinación de las variables zoométricas: índice cefálico (ICF), índice de proporcionalidad (IPD), índice corporal (ICP), índice pelviano (IPV), índice torácico (ITO), profundidad relativa del pecho (PRP), índice metacarpo torácico (IMT), índice facial (IF) e índice de carga de la caña (ICC). En el programa MSTAT-C (1998), se analizaron los siguientes estadísticos descriptivos: la media como valor de tendencia central, desviación estándar (DE) y coeficiente de variación $(\mathrm{CV})$ como estadísticos dispersivos. Así mismo, se registraron ocho variables fanerópticas de tipo cualitativo: perfil frontonasal, color de capa, color de mucosas, color de pezuñas, ausencia/ presencia de pelo, orientación de las orejas, ausencia/ presencia de mamellas y tipo de cerdo; las cuales fueron analizadas a través de la prueba no paramétrica de chi cuadrado $\left(\mathrm{X}^{2}\right)$.

\section{Resultados y Discusión}

$E^{1}$ análisis de varianza entre localidades (Cuadro 1) reportó resultados muy homogéneos, a excepción de la anchura de cabeza (ACZ), anchura de hocico $(\mathrm{AH})$ que fueron superiores en la localidad de La Maná $(\mathrm{P}<0.01)$ y la longitud de grupa $(\mathrm{LGR})$ que fue mayor en la localidad de Valencia $(\mathrm{P}<0.05)$. El peso vivo promedio fue de 58.21 y $59.50 \mathrm{~kg}$ en Valencia y La Maná, respectivamente, presentando una gran variabilidad con un CV de 36.92 y $35.93 \%$; información similar a la de Revidatti et al. (2005) que reportó un peso promedio de $60.47 \mathrm{~kg}$ con CV de $41 \%$ en una población de cerdos caracterizados en la provincia de Corrientes, Argentina. Las variable ALC presentó medias de $60.99 \mathrm{~cm}$ en Valencia y 61.74 en La Maná, similares a las reportadas por Barba et al. (1998) en la caracterización de cerdos criollos cubanos. El DL y PTO registraron valores promedios de 80.12 y $93.68 \mathrm{~cm}$ en Valencia y 79.12 y 92.50 $\mathrm{cm}$, en La Maná, con gran semejanza a los obtenidos por Hurtado et al. (2005), quienes realizaron el estudio morfológico del cerdo criollo del estado de Apure en Venezuela. La LGR y AGR evidenciaron promedios de 30.08 y $23.08 \mathrm{~cm}$ en Valencia, 28.32 y $22.26 \mathrm{~cm}$ en La Maná.

Los resultados de los índices zoométricos por localidad se detallan en el Cuadro 2. Entre localidades, los índices de proporcionalidad (IDP), corporal (ICP), toráxico (ITO), profundidad relativa del pecho (PRP), facial (IF) y de carga de la caña (ICC) no presentaron diferencias; a excepción del índice cefálico (ICF) que fue superior en La Maná con $51.15 \%$ vs $45.89 \%$ en Valencia $(\mathrm{P}<0.01)$, valores mayores a los reportados por Revidatti et al. (2004) en un estudio de los índices zoométricos en el cerdo criollo de la región nordeste 
argentina, indicando que se trata de una población de alargado (dolicocéfalo) en Valencia.

cráneo muy alargado (hiperdolicocéfalo) en La Maná y

Cuadro 1. Estadísticos descriptivos del peso vivo (kg) y variables zoométricas (cm) de una población de cerdos criollos en los cantones Valencia y La Maná. UICYT-UTEQ. 2007

\begin{tabular}{lrrrrrrr}
\hline \multirow{2}{*}{\multicolumn{1}{c}{ Variables }} & \multicolumn{3}{c}{ Valencia $(\mathbf{n}=\mathbf{6 1})$} & \multicolumn{3}{c}{ La Maná (n=88) } \\
\cline { 2 - 8 } & Media & \multicolumn{1}{c}{ DE } & CV (\%) & Media & \multicolumn{1}{c}{ DE } & CV (\%) & Sig. \\
\hline Peso vivo (PV, kg) & 58.21 & 21.49 & 36.92 & 59.50 & 21.38 & 35.93 & NS \\
Longitud cabeza (LCZ, cm) & 28.09 & 3.27 & 11.63 & 28.85 & 4.52 & 15.66 & NS \\
Anchura cabeza (ACZ, cm) & 12.70 & 1.76 & 13.88 & 14.64 & 2.70 & 18.45 & $* *$ \\
Longitud hocico (LH, cm) & 17.09 & 2.71 & 15.86 & 17.50 & 3.96 & 22.67 & NS \\
Anchura hocico (AH, cm) & 8.62 & 1.33 & 15.43 & 10.11 & 2.67 & 26.46 & $* *$ \\
Longitud de grupa (LGR, cm) & 30.08 & 3.12 & 10.37 & 28.32 & 4.66 & 16.46 & $*$ \\
Anchura grupa (AGR, cm) & 23.08 & 2.85 & 12.38 & 22.26 & 4.37 & 19.62 & NS \\
Alzada cruz (ALC, cm) & 60.99 & 8.74 & 14.33 & 61.74 & 11.24 & 18.21 & NS \\
Alzada grupa (ALG, cm) & 67.63 & 9.26 & 13.69 & 67.98 & 11.03 & 16.22 & NS \\
Alzada nacimiento cola (ANC, cm) & 53.80 & 7.25 & 13.48 & 53.54 & 9.17 & 17.14 & NS \\
Diámetro longitudinal (DL, cm) & 80.12 & 11.04 & 13.78 & 79.12 & 12.92 & 16.33 & NS \\
Diámetro dorsoesternal (DDE, cm) & 37.49 & 6.12 & 16.33 & 37.51 & 9.51 & 25.35 & NS \\
Diámetro bicostal (DBC, cm) & 25.12 & 5.14 & 20.47 & 24.58 & 5.59 & 22.77 & NS \\
Perímetro toráxico (PTO, cm) & 93.68 & 14.37 & 15.34 & 92.50 & 17.95 & 19.41 & NS \\
Perímetro caña (PCA, cm) & 14.68 & 2.12 & 14.46 & 15.36 & 2.11 & 13.77 & NS \\
Longitud oreja (LO, cm) & 20.35 & 3.52 & 17.30 & 19.48 & 4.27 & 21.93 & NS \\
Anchura oreja (AO, cm) & 17.31 & 2.69 & 15.56 & 17.80 & 3.13 & 17.59 & NS \\
\hline
\end{tabular}

$\mathrm{NS}=$ No significativo, $*=$ Significativo, $* *=$ Altamente significativo

$\mathrm{DE}=$ Desviación estándar, $\mathrm{CV}=$ Coeficiente de variación

El índice pelviano (IPV) en La Maná fue de 79.69 vs $76.67 \%$ en Valencia $(\mathrm{P}<0.05)$, promedios inferiores a $83.04 \%$ registrado por Hurtado et al. (2005).
También, se pudo observar que el índice metacarpo toráxico (IMT) en La Maná fue de 16.69 vs $15.77 \%$ en Valencia $(\mathrm{P}<0.01)$.

Cuadro 2. Estadísticos descriptivos de los índices zoométricos (\%) de una población de cerdos criollos en los cantones Valencia y La Maná. UICYT-UTEQ. 2007

\begin{tabular}{lccccccc}
\hline \multirow{2}{*}{\multicolumn{1}{c}{ Índices zoométricos (\%) }} & \multicolumn{3}{c}{ Valencia $(\mathbf{n = 6 1 )}$} & \multicolumn{3}{c}{ La Maná (n=88) } \\
\cline { 2 - 8 } & Media & DE & CV (\%) & Media & DE & CV (\%) & Sig. \\
\hline Índice cefálico (ICF) & 45.89 & 6.68 & 14.57 & 51.15 & 7.94 & 15.52 & $* *$ \\
Índice proporcionalidad (IPD) & 76.41 & 7.36 & 9.64 & 78.80 & 13.58 & 17.23 & NS \\
Índice corporal (ICP) & 85.60 & 6.76 & 7.90 & 87.07 & 13.45 & 15.45 & NS \\
Índice pelviano (IPV) & 76.67 & 6.85 & 8.93 & 79.69 & 8.15 & 10.23 & $*$ \\
Índice toráxico (ITO) & 67.20 & 8.75 & 13.02 & 67.01 & 11.91 & 17.77 & NS \\
Profundidad relativa pecho (PRP) & 61.69 & 7.30 & 11.83 & 61.48 & 11.36 & 18.48 & NS \\
Índice metacarpo toráxico (IMT) & 15.77 & 1.47 & 9.31 & 16.69 & 2.19 & 13.16 & $* *$ \\
Índice facial (IF) & 60.92 & 6.85 & 11.24 & 60.32 & 9.63 & 15.97 & NS \\
Índice de carga de la caña (ICC) & 27.58 & 7.52 & 27.26 & 28.65 & 9.14 & 31.92 & NS \\
\hline
\end{tabular}

$\mathrm{NS}=$ No significativo,$*=$ Significativo, $* *=$ Altamente significativo

$\mathrm{DE}=$ Desviación estándar, $\mathrm{CV}=$ Coeficiente de variación 
Las variables fanerópticas se presentan en el Cuadro 3. Se observó con mayor frecuencia el perfil frontonasal recto (85.25 y 73.86\%), en Valencia y La Maná (Foto 1). El color de capa y mucosa presentaron diferencias significativas entre localidades $\left(\mathrm{X}^{2}=12.27\right.$ y 10.54, respectivamente), en Valencia sobresalió la capa colorada con $32.79 \%$ y, en La Maná la manchada con $30.68 \%$. En la zona de Valencia destacó la mucosa oscura con $40.98 \%$ y la clara en La Maná (Foto 2) con $37.50 \%$. El mayor porcentaje de los animales presentaron pelo (Foto 3); sobre la orientación de las orejas predominan las tipo tejas o ibéricas (Foto 4) en Valencia (49.18\%) y en La Maná (43.18\%).

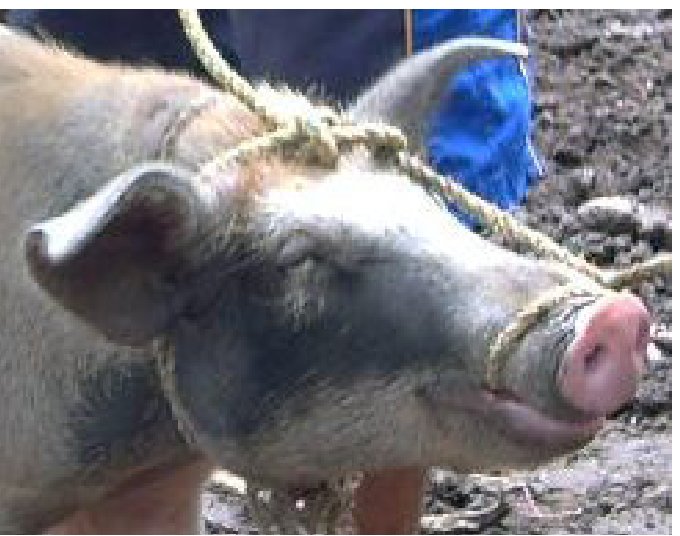

Foto 2. Cerdo criollo de la zona de La Maná con mucosa clara.

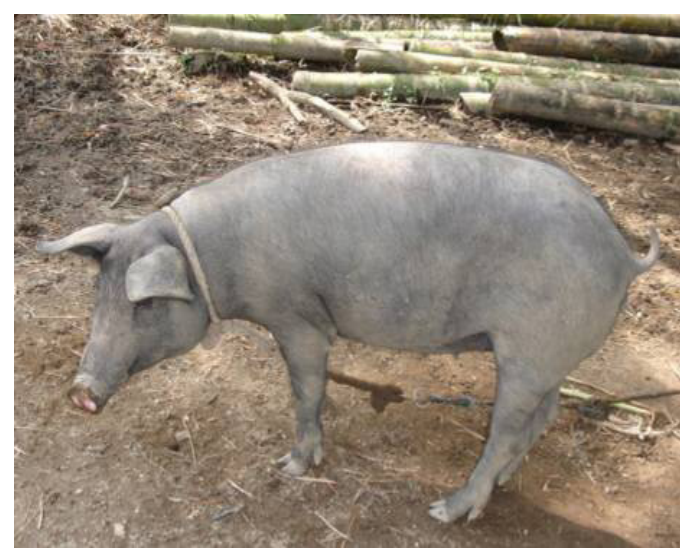

Foto 4. Cerda criolla de la zona de Valencia con orejas tipo ibéricas. 
Cuadro 3. Frecuencias absoluta y relativa (\%) de las variables cualitativas en hembras y machos de una población de cerdos naturalizados en los cantones Valencia y La Maná. UICYT-UTEQ. 2007

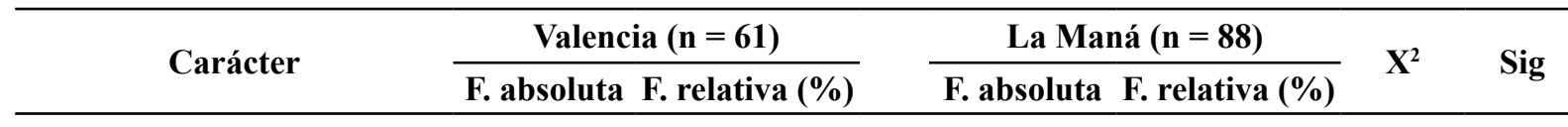

\section{P. frontonasal}

1. Recto

2. Cóncavo

3. Subcóncavo

\section{Color de capa}

1. Colorada

2. Overa o baya

3. Negra pizarra

4. Manchada

5. Otras ${ }^{1}$

\section{Color mucosa}

1. Clara

2. Oscura

3. Manchada

4. Despigmentada

Color pezuñas

1. Blanca

2. Negra

3. Veteada

4. Otras ${ }^{2}$

Pelo

1. Abundante

2. Escaso

3. Lampiño

\section{Orientación Orejas}

1. Erectas

2. Tejas

3. Caídas

Mamellas

1. Presencia

2. Ausencia

Tipo de cerdos

1. Magro

2. Graso

3. Doble propósito

$\mathrm{X}^{2}=$ Prueba de chi cuadrado

NS $=$ No significativo, $*=$ Significativo, $* *=$ Altamente significativo

${ }^{1}$ Blanca con manchas negras, negra con vetas blancas, blanca

${ }^{2}$ Blanco y negro
52

8

1

20

0

14

16

11

17

25

11

8

31

12

18

0

33

28

0

54.10

45.90

0.00

13.11

49.18

37.71

14.75

85.25

22.95

4.92

72.13

52
73.86

22.73

3.41

$3 \quad 3.41$

\section{5}

11

17.04

12.50

23

26.14

27

30.68

12

13.64
$2.78 \quad \mathrm{NS}$

33

37.50

36.36

32

25.00

22

1.14

$1 \quad 1.14$

20

22.73

31.82

28

39.77

35

5.68

5

55

62.50

28.41

$\begin{array}{rr}25 & 28.41 \\ 8 & 9.09\end{array}$

8

26

29.54

43.18

38

24

27.28

14.81

10.54 *

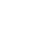

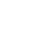

7.95

$1.10 \mathrm{NS}$

81

92.05 


\section{Conclusiones}

Qe registró una gran variabilidad de los pesos de los animales adultos, con coeficientes de variación de $36.92 \%$ en la zona de Valencia y $35.93 \%$ y en La Maná.

El análisis de varianza de las localidades no presentó diferencias estadísticas en la mayoría de las variables zoométricas, a excepción de anchura de cabeza (ACZ), anchura de hocico (AH) que fueron superiores en La Maná $(\mathrm{P}<0.01)$ y longitud de grupa que fue mayor en Valencia $(\mathrm{P}<0.05)$.

El índice cefálico (ICF) de la zona de Valencia de $45.15 \%$ y de la zona de La Maná $51.15 \%$; demostrando una tendencia de la muestra en la primera localidad de cráneo muy alargado "hiperdolicocéfalo" y en la segunda el cráneo es alargado "dolicocéfalo".

Las características fanerópticas son muy variables, se observó mayor frecuencia del perfil frontonasal recto en Valencia y La Maná. El color de la capa fue heterogéneo. Se evidenció abundancia de pelo y en la orientación de las orejas predominan las ibéricas en ambas localidades.

\section{Literatura CitADA}

Aparicio, G. 1960. Zootecnia Especial. Córdoba, España. pp. 5-27; 451-467.

Barba, C., A. Cabello, R. Sanz y J. Delgado. 2004. Programa demostrativo de técnicas en caracterización productiva y morfológica. En: Delgado, J. V. (Ed.). Biodiversidad Porcina Iberoamericana: Caracterización y Uso Sustentable. pp. 303-311.

Barba, C., F. Velásquez, F. Rodríguez, F. Pérez, J. Delgado. 1998. Contribución al estudio racial del cerdo criollo cubano. Arch. Zootec. 47: 51 - 59.

Delgado, J., M. E. Camacho, J. M. León, M. R. de la Haba, A. Vallecillo, C. Barba, A. Cabello. 2004. En: Delgado, J. V. (Ed). Biodiversidad porcina Iberoamericana: Poblaciones porcinas de Iberoamérica pp. 21 - 29.
Delgado, J., J. Vega, C. Barba, A. Martinez, M. Zamorano. 1998. Caracterización morfológica y genética de las variedades del tronco ibérico. Solo Cerdo Ibérico 1: 27.

Estupiñán, K., D. Vasco, S. Barreto y K. Zambrano. 2007. Estudio morfoestructural de una población de cerdos naturalizados en el cantón La Maná, provincia de Cotopaxi, Ecuador. Memorias VIII Simposio Iberoamericano sobre Conservación y Utilización de Recursos Zoogenéticos. pp. 166174.

Hurtado, E., C. González e H. Vecchionacce. 2005. Estudio morfológico del cerdo criollo del estado Apure, Venezuela. Zootecnia Trop. 23: 17- 26.

MSTAT-C. 1998. Microcomputer Program for the Design Management and Análisis of Agronomic Research Experiments. Michigan State University.

Revidatti, M., A. Capellari, P. Prieto, J. Delgado, G. Rebak. 2004. Índices zoométricos en el cerdo criollo de la Región Nordeste Argentina. Memorias V Simposio Iberoamericano sobre Conservación y utilización de Recursos Zoogenéticos. pp. 112115.

Revidatti, M., J. Delgado, A. Capellar y P. Prieto. 2005. Estudio morfoestructural preliminar de una población porcina en la provincia de Corrientes, Argentina. Arch. de Zootec. 54: 227-232.

Sanz, R., E. Dieguez y A. Cabello. 2004. Caracterización morfológica, productiva y reproductiva de las variedades del cerdo Ibérico. En: Delgado, J. V. (Ed). Biodiversidad porcina Iberoamericana. Córdoba - España. pp. 209 - 217.

Sotillo, J. y V. Serrano. 1985. Producción Animal. Etnología Zootécnica. Tomo I. Albacete, España. 403 p. 